\title{
Isobologram Curve of Leishmanicidal Effects of Amiodarone and
} Ketoconazole

\author{
Somayeh Bahrami, ${ }^{1,}{ }^{*}$ Mohammad Hossein Razi Jalali, ${ }^{1}$ Hossein Najafzadeh, ${ }^{2}$ and Zeinab Asadi ${ }^{1}$ \\ ${ }^{1}$ Department of Pathobiology, Faculty of Veterinary Medicine, Shahid Chamran University of Ahvaz, Iran \\ ${ }^{2}$ Department of Pharmacology and Toxicology, Faculty of Veterinary Medicine, Shahid Chamran University of Ahvaz, Iran \\ "Corresponding author: Somayeh Bahrami, Department of Pathobiology, Faculty of Veterinary Medicine, Shahid Chamran University of Ahvaz, Iran. Tel: +98-6113738636, Fax: \\ +98-6113360807, E-mail: s.bahrami@scu.ac.ir \\ Received 2016 October 10; Revised 2016 December 05; Accepted 2017 February 04.
}

\begin{abstract}
Background: Leishmaniasis is a zoonotic parasitic infection with a worldwide distribution. Ergosterol and stigmasterol, equivalent to mammalian cholesterol, are the key sterols in Leishmania species, and thus can be used as proper targets for drugs. Objective: Because amiodarone (AMD) and ketoconazole (KTZ) inhibit the sterol biosynthesis in trypanosomatid parasites, the present study aimed at investigating the effects of AMD and KTZ, acting individually or in combination, against L. infantum promastigotes and axenic amastigotes.

Methods: For each stage, drugs were added to the growth medium 24 hours after starting the cultures with $10^{6}$ parasites $/ \mathrm{mL}$. Replication of L. infantum promastigotes and axenic amastigotes were investigated in the presence and absence of AMD and KTZ. Isobologram construction and calculation of the FIC were performed.

Results: Minimum concentration, which produces total death of parasites against promastigotes and axenic amastigotes, was 28 $\mu \mathrm{M}$ and $12 \mu \mathrm{M}$ for AMD and $1 \mu \mathrm{M}$ and $0.75 \mu \mathrm{M}$ for KTZ. According to the concave isobologram and fractional inhibitory concentration $<1$, antiproliferative synergism of the drugs was confirmed.

Conclusions: Both drugs in synergy could be effective in arresting parasite proliferation at doses lower than those required when used alone, reducing the potential cost (KTZ) or toxic effects (AMD) involved.
\end{abstract}

Keywords: Amiodarone, Ketoconazole, Isobologram Curve, Leishmania Infantum

\section{Background}

Leishmania parasites are obligatory intracellular parasites that attack macrophages in the mammalian hosts and their transmission happens through arthropods phlebotomine sandflies. In several regions, leishmaniasis is considered among the major zoonotic diseases, affecting hygiene (1). Leishmaniasis has an expanded clinical manifestation, from the cutaneous lesions that cure spontaneously to the possibly mortal visceral leishmaniasis, in which parasites propagate from the site of insect bites and have a tropism for the mononuclear phagocytic system, especially in the liver and spleen (2). Also, subclinical infection is detected in asymptomatic immunocompetent individuals. The overall worldwide prevalence of leishmaniasis is 12 million people. The global incidence of all clinical forms occurs in 2 million new cases annually (3). Leishmaniasis is widespread in the Mediterranean areas and in Iran $(4,5)$. In Iran, visceral leishmaniasis frequently occurs in children younger than 2 years (6) and a wide spectrum of clinical manifestations are reported, particularly in immunocompromised patients (7). Weight loss, fever, cough, and spleen and liver enlargement are visceral leishmani- asis symptoms. Furthermore, more than $90 \%$ of patients who receive no medication will die (8).

There are different classical drugs like sodium meglumine antimonite (glucantime), stibogluconte (pentostam), amphotericin B, paramomycin, desoxycholate, and lipid formulations of amphotericin B, miltefosine, which have been employed as effective medications of visceral leishmaniasis for decades (9). However, a complete treatment of leishmaniasis is often laborious due to expense, toxic side effects, and increasing resistance (10). Thus, developments of new efficacious chemotherapeutic agents are important for the disease. Ergosterol is a sterol found in cell membranes of Leishmania and pathogenic fungi and its synthesis, and incorporation into cell membranes are necessary for growth and proliferation. Many azole antifungal agents suppress the cytochrome P450-enzyme lanosterol 14- $\alpha$ demethylase activity, which is responsible for the production of ergosterol from lanosterol (11). On the other hand, selective activity of amiodarone (AMD), the antiarrhythmic drug, has been claimed against some species of Leishmania such as L. Mexicana, L. amazonensis, and Tryoanosoma cruzi. One of the mechanisms of action that AMD induced in different 
pathogens was inhibition of sterol biosynthesis (12-14).

\section{Objectives}

Because targeting the ergosterol synthesis pathway of Leishmania may be useful therapeutically, in this work we aimed at investigating the effects of AMD on L. infantum and its potential synergistic effects with ketoconazole (KTZ).

\section{Methods}

\subsection{Drugs}

Amiodarone ((2-butyl-3-benzofuranyl)- (4- (2- (diethylamino) ethoxi)-3, 5-diiodophenyl) methanone hydrochloride) and ketoconazole (-1-(4- (4- (-2- (2,4-Dichlorphenyl) 2- (imidazol-1-ylmethyl) -1,3-dioxolan-4-ylmethoxy) phenyl piperazin-1-yl) ethanon) were prepared from Sigma Aldrich (St. Louis, MO).

\subsection{Promastigotes and Axenic Amastigotes Culture}

Leishmania infantum (MCAN/IR/07/Moheb-gh) promastigotes were grown at $26^{\circ} \mathrm{C}$ in brain-heart infusion (BHI) medium plus $10 \%$ heat-inactivated fetal calf serum (FCS), pH7.0, and $1 \%$ of penicillin $(50 \mathrm{U} / \mathrm{mL}$ ) streptomycin $(50 \mu \mathrm{g} / \mathrm{mL})$ solution (Sigma, St. Louis, MO, USA). Temperature status and parasites $\mathrm{pH}$ in macrophage phagolysosomes had the pattern of L. infantum promastigotes transformation to axenic amastigotes $(15,16)$. The cultures were initiated by $5 \mathrm{~mL}$ of the medium with a concentration of $5 \times 10^{6}$ stationary-phase promastigotes $/ \mathrm{mL}$ in $25 \mathrm{~cm}^{2}$ tissue flasks. For L. infantum, a high proportion (73\%) of the amastigote- like forms was obtained in BHI medium supplemented with $20 \%$ FCS at pH 5.5 after 48 hours of incubation at $37^{\circ} \mathrm{C}$ in the presence of $5 \% \mathrm{CO}_{2}$. Before counting, the parasites were passed through a 25 gauge needle to disrupt the aggregated forms in the cultures, and parasite number was calculated using the Neubauer chamber. Three criteria including shape, lack of flagellum, and viability were checked every 12 hours to evaluate the transformation of promastigotes to axenic amastigotes. The viability of axenic amastigotes was evaluated by both Trypan blue (vital dye) and their differentiation back to promastigotes by shifting them to grow at $26^{\circ} \mathrm{C}$ in $\mathrm{pH}$ 7.0. To evaluate the morphology of axenic amastigotes, parasites were collected from the cultures, centrifuged with $50 \mathrm{mM}$ phosphate buffered saline (PBS) at $400 \mathrm{~g}$, and applied to microscope slides. The slides were stained by Giemsa and examined by a light microscope (Olympus Optical, NY, model BX60, Tokyo, Japan) at x1000 magnification.
3.3. Effects of Ketoconazole and/or Amiodarone on Promastigotes and Axenic Amastigotes

Drugs were added 24 hours after starting the cultures with $10^{6}$ parasites $/ \mathrm{mL}$. Susceptibility of L. infantum promastigotes and axenic amastigotes to AMD and KTZ was evaluated by following up the proliferation of parasites in the absence or presence of these drugs, counting living parasites daily in a Neubauer chamber during 48 hours of growth. At least 3 independent experiments were done for each drug or combined mode.

\subsection{Calculation of Fractional Inhibitory Concentration (FIC) and Isobologram Curve Construction}

Isobologram construction and determination of the FIC were performed based on the methods described by Hallander et al. (1982) and Reimao and Tempone (2011) (17, 18). FIC was calculated as the combined concentration divided by single concentration. The interaction index was calculated as follows:

$\sum$ FIC $=($ MIC combination A/MIC alone $\mathrm{A})+($ MIC com bination $\mathrm{B} / \mathrm{MIC}$ alone $\mathrm{B}$ ), where MIC is the MIC that produces total death of parasite population in the culture, with A representing the AMD concentration and B the KTZ concentration.

\section{Results}

Leishmania infantum promastigotes and axenic amastigotes were treated with different concentrations of AMD and KTZ, and their proliferation was followed during 2 days. Based on the results, treatment demonstrated a concentration-dependent inhibition of the proliferation. For promastigotes and axenic amastigotes, 50\% inhibitory concentration of AMD achieved $6 \mu \mathrm{M}$ and 5.2 $\mu \mathrm{M}$, respectively, after 48 hours of treatment. Inhibition of around $100 \%$ was obtained with a concentration of 28 $\mu \mathrm{M}$ and $12 \mu \mathrm{M}$ for promastigotes and axenic amastigotes, respectively. Ketoconazole also induced a dose-dependent reduction of both promastigotes and axenic amastigotes proliferation with an IC50 value of $0.4 \mu \mathrm{M}$ and $0.34 \mu \mathrm{M}$ and MIC $1 \mu \mathrm{M}$ and $0.75 \mu \mathrm{M}$, respectively. Table 1 displays IC50 and MIC of AMD and KTZ for promastigotes and axenic amastigotes of L. infantum.

To evaluate the effect of the combined drugs and their interactions, isobologram curves were established from the MICs achieved for the drugs used alone or in combination.

Based on the concave and FIC value of 0.51 and 0.42 for promastigotes and axenic amastigotes, the results revealed a synergistic interaction when the AMD and KTZ were used in combination (Figure 1). 
Table 1. Showed IC50 and MIC ( $\mu \mathrm{M})$ of AMD and KTZ for Promastigotes and Axenic Amastigotes of L. Infantum

\begin{tabular}{|c|c|c|c|c|}
\hline \multirow[t]{2}{*}{ Stage of L. Infantum } & \multicolumn{2}{|c|}{ Amiodarone } & \multicolumn{2}{|c|}{ Ketoconazole } \\
\hline & IC50 & MIC & IC50 & MIC \\
\hline Promastigotes & 6 & 28 & 0.4 & 1 \\
\hline Axenic amastigotes & 5.2 & 12 & 0.34 & 0.75 \\
\hline
\end{tabular}

Figure 1. Representative Isobologram of in Vitro Interaction Between Amiodarone and Ketoconazole Against L. Infantum Promastigotes (A) and Axenic Amastigotes (B)

A

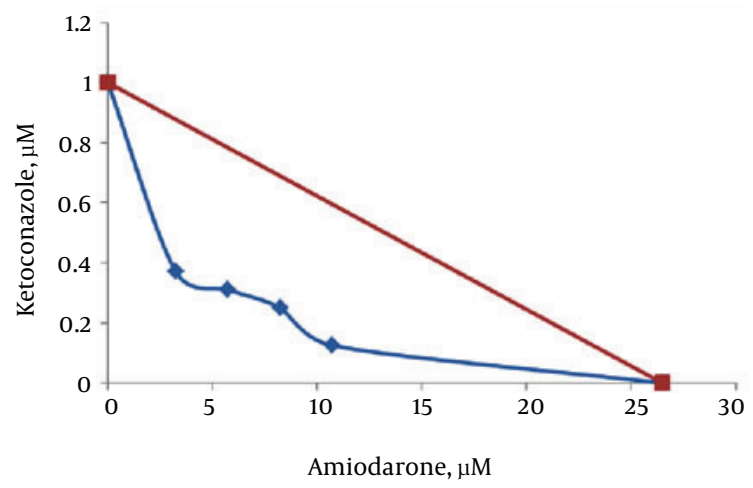

B

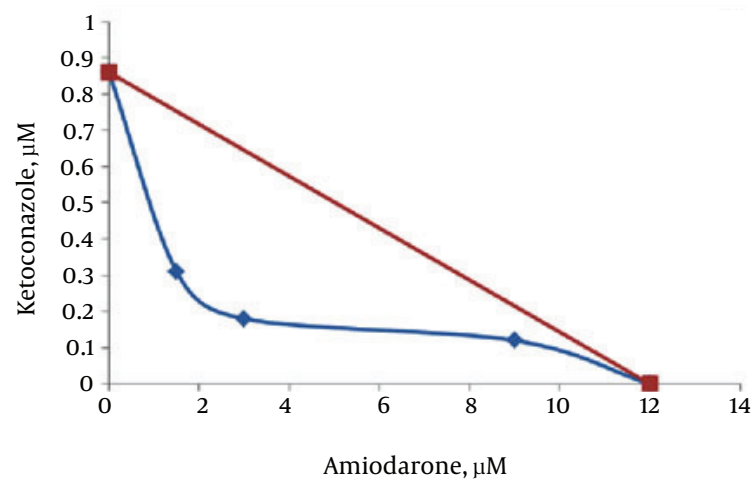

The dotted lines correspond to the predicted positions of the experimental points for a simple additive effect. Concave isoboles represent synergistic effect of amiodarone and ketoconazole for promastigotes and axenic amastigotes.

\section{Discussion}

In most cases, pentavalent antimonials remain an efficient antileishmanial treatment, but their parenteral route of administration, long duration of treatment, and side effects limit their usages (19). In patients with human immunodeficiency virus, the antimonial drugs are inefficient and toxic (20). Moreover, the emergences of antimony resistant strains and the increase in the number of therapeutic failures have been reported. In the case of resistance to pentavalent antimonials, amphotericin B or pentamidine as the second- line treatments have been successfully used (21). In India, oral consumption of miltefosine is a first-line treatment for visceral leishmaniasis (22); however, its teratogenicity should be considered (23). More recently, combination treatments are suggested as first-line treatments for visceral leishmaniasis (24).

Normal function and structure sterols are necessary for cellular membrane. Cholesterol is the principal sterol in different membranes of mammalians. In the past, it was thought that cholesterol was the major sterol for trypanosomatids (25). Thereafter, it was determined that the trypanosomatids obtain cholesterol from the blood of infected animals or from the culture medium through a typical endocytic process (26). In these organisms, sterol biosynthesis is a key metabolic pathway, which produces different sterols including ergosterol and other 24-methyl sterols, that is, essential for viability and growth $(27,28)$.

Amiodarone as the antiarrhythmic class III drug has generally been used to treat arrhythmias. Also, it is suggested for the treatment of patients with cardiac compromise and chronic Chagas' disease. Inactivation of $\mathrm{Ca}^{2+}$ and $\mathrm{K}^{+}$channel is the mechanism of its antiarrhythmic action in mammals. Also, antiparasitic effects of AMD have been claimed $(12,13,29)$ as well as a broad-spectrum antifungal action (30). Interfering with mitochondrial membrane potential $(\Delta \Psi \mathrm{m})$, inhibition of sterol biosynthesis, $\mathrm{Ca}^{2+}$ homeostasis disordering as well as reactive oxygen species production are the main mechanisms of action of AMD against different organisms (12, 13, 30-32). Mitochondrial metabolism alterations induce parasites apoptosis (32). On the other hand, the drugs with azole group such as ketoconazole are proper for fungal infections treatment. Also, they inhibit proliferation of different Leishmania spp and T. cruzi in vitro. Blockage of the parasite cytochrome P450dependent C14-demethylase (CYP51), $14 \alpha$-methylsterols elevation, and ergostane-derived sterols decline are the proposed action mechanism of AMD (28). Based on this information, the effect of the combination of AMD and KTZ was 
evaluated in the present study.

Benaim et al. (2006) stated that AMD has a significant activity against T. cruzi (12). Veiga-Santos et al. (2012) confirmed that AMD possesses intrinsic activity against the proliferation of T. cruzi epimastigote and amastigote without affecting the host cell viability (33). de Macedo-Silva et al. (2011) also found mitochondrion- kinetoplast complex alterations and large autophagosomes in L. amazonensis promastigotes incubated with AMD (14). In Serrano-Martin et al. study (2009), it was demonstrated that amiodarone causes squalene accumulation in L. mexicana promastigotes by the biosynthesis of 5-dehydroepisterol and inhibition of squalene epoxidase activity (13).

Some studies have claimed that ketoconazole has leishmanicidal effects (34). Lazardi et al. (1990) stated the presence of autophagic vacuoles in amastigotes treated with ketoconazole (35). According to Wagner and UlrichMerzenich (2009), synergism between 2 drugs can be demonstrated when the effect produced by the compounds used in combination is higher than the sum of the effects of each compound used alone. Interactions between 2 drugs are calculated geometrically or algebraically. In the latter method, the concentration of each drug in the combination that produces the intended effect is expressed as a fraction of the concentration that leads to the same effect when the drug is used alone, i.e., its fractional inhibitory concentration. The combination of drugs is additive when the total of fractions is 1 , the combination is synergistic when the total is lower than 1 , and the combination is antagonistic when the total is more than 1 . In the geometrical method, a curve is prepared with the axes considering drug concentrations on linear scales. When the line connecting the points that shows all combinations with the same result (including the same effective concentrations of the drugs used alone) is straight, the isobole shows that the combination has an additive effect. Synergistic combinations give concave isoboles, while the convex isoboles demonstrates antagonistic effects (36). In the present study, the data led to a concave isobologram and the FIC value of 0.51 obtained for promastigotes and 0.42 for axenic amastigotes indicated clear synergism. In Benaim et al. study (2006), the synergistic effects between AMD and posaconazole have been demonstrated against $T$. cruzi amastigotes (12). In Veiga-Santos et al. study (2012), it was found that the combination of AMD and posaconazole exhibit potent synergistic activity against T. cruzi (33). In Serrano Martin et al. (2009) investigation, AMD and miltefosine combination had synergistic inhibitory effects on the proliferation of intracellular amastigotes (29). Results of the present study indicate that AMD and KTZ act synergistically gainst L. infatum for the first time. Due to the intrinsic activity of AMD against kinetoplastid parasites, it seems that this drug could be consumed simultaneously in combination with antiprotozoal drugs to treat parasitic infections at low doses, leading to decrease its side effects such as cardiotoxicity, thyroid impairment, and pulmonary fibrosis. In conclusion, this study revealed that the combination of amiodarone plus ketoconazole acts synergistically for inhibition of L. infantum proliferation in vitro. Both drugs are commercially available, becoming candidates for the treatment of patients with leishmaniasis.

\section{Acknowledgments}

This study was supported by the research grant provided by Shahid Chamran University of Ahvaz.

\section{Footnotes}

Authors' Contribution: Study concept and design, Somayeh Bahrami; data acquisition, Mohammad Hossein Razi Jalali; data analysis and interpretation, Hossein Najafzadeh; drafting the manuscript, Zeinab Asadi; critical revision of the manuscript in terms of intellectual content, Somayeh Bahrami; statistical analysis, Somayeh Bahrami; administrative, technical, and material support, Somayeh Bahrami and Hossein Najafzadeh; study supervision, Somayeh Bahrami.

Funding/Support: This study was financially supported by a grant from the research council of Shahid Chamran University of Ahvaz, Iran.

\section{References}

1. Murray HW, Berman JD, Davies CR, Saravia NG. Advances in leishmaniasis. Lancet. 2005;366(9496):1561-77. doi: 10.1016/S01406736(05)67629-5. [PubMed: 16257344].

2. Simonsen PE, Cook GC, Zumla AI. Manson's tropical diseases. Elsevier Health Sciences; 2009.

3. Choi CM, Lerner EA. Leishmaniasis as an emerging infection. J Investig Dermatol Symp Proc. 2001;6(3):175-82. doi: 10.1046/j.0022202x.2001.00038.x. [PubMed: 11924824].

4. Saki J, Meamar A, Oormazdi H, Akhlaghi L, Maraghi S, Mohebali $\mathrm{M}$, et al. Mini-exon genotyping of leishmania species in khuzestan province, southwest iran. Iran J Parasitol. 2010;5(1):25-34. [PubMed: 22347232].

5. Saki J, Khademvatan S. A molecular study on cutaneous leishmaniasis lesions in Khuzestan province (South west of Iran). Jundishapur J Microbiol. 2011;4(4):283-8.

6. Alborzi A, Rasouli M, Shamsizadeh A. Leishmania tropica-isolated patient with visceral leishmaniasis in southern Iran. Am J Trop Med Hyg. 2006;74(2):306-7. [PubMed: 16474088].

7. Mohebali M, Hajjaran H, Hamzavi Y, Mobedi I, Arshi S, Zarei Z, et al. Epidemiological aspects of canine visceral leishmaniosis in the Islamic Republic of Iran. Vet Parasitol. 2005;129(3-4):243-51. doi: 10.1016/j.vetpar.2005.01.010. [PubMed: 15845279]. 
8. Di Giorgio C, Delmas F, Ollivier E, Elias R, Balansard G, TimonDavid P. In vitro activity of the beta-carboline alkaloids harmane, harmine, and harmaline toward parasites of the species Leishmania infantum. Exp Parasitol. 2004;106(3-4):67-74. doi: 10.1016/j.exppara.2004.04.002. [PubMed: 15172213].

9. Rosenthal E, Marty P. Recent understanding in the treatment of visceral leishmaniasis. J Postgrad Med. 2003;49(1):61-8. [PubMed: 12865573].

10. Savoia D. Recent updates and perspectives on leishmaniasis. I Infect Dev Ctries. 2015;9(6):588-96. doi: 10.3855/jidc.6833. [PubMed: 26142667].

11. Korashy HM, Shayeganpour A, Brocks DR, El-Kadi AO. Induction of cytochrome P450 1A1 by ketoconazole and itraconazole but not fluconazole in murine and human hepatoma cell lines. Toxicol Sci. 2007;97(1):32-43. doi:10.1093/toxsci/kfm012. [PubMed: 17283379].

12. Benaim G, Sanders JM, Garcia-Marchan Y, Colina C, Lira R, Caldera AR, et al. Amiodarone has intrinsic anti-Trypanosoma cruzi activity and acts synergistically with posaconazole. J Med Chem. 2006;49(3):8929. doi:10.1021/jm050691f. [PubMed: 16451055].

13. Serrano-Martin X, Garcia-Marchan Y, Fernandez A, Rodriguez N, Rojas $\mathrm{H}$, Visbal G, et al. Amiodarone destabilizes intracellular Ca2+ homeostasis and biosynthesis of sterols in Leishmania mexicana. Antimicrob Agents Chemother. 2009;53(4):1403-10. doi: 10.1128/AAC.01215-08. [PubMed: 19164149].

14. de Macedo-Silva ST, de Oliveira Silva TL, Urbina JA, de Souza W, Rodrigues JC. Antiproliferative, Ultrastructural, and Physiological Effects of Amiodarone on Promastigote and Amastigote Forms of Leishmania amazonensis. Mol Biol Int. 2011;2011:876021. doi: 10.4061/2011/876021. [PubMed: 22091415].

15. Bahrami S, Hatam GR, Razavi M, Nazifi S. In vitro cultivation of axenic amastigotes and the comparison of antioxidant enzymes at different stages of Leishmania tropica. Trop Biomed. 2011;28(2):411-7. [PubMed: 22041763].

16. Gupta N, Goyal N, Rastogi AK. In vitro cultivation and characterization of axenic amastigotes of Leishmania. Trends Parasitol. 2001;17(3):1503. doi: 10.1016/S1471-4922(00)01811-0. [PubMed: 11286801].

17. Hallander HO, Dornbusch K, Gezelius L, Jacobson K, Karlsson I. Synergism between aminoglycosides and cephalosporins with antipseudomonal activity: interaction index and killing curve method. Antimicrob Agents Chemother. 1982;22(5):743-52. doi:10.1128/AAC.22.5.743. [PubMed: 7181485].

18. Reimao JQ, Tempone AG. Investigation into in vitro anti-leishmanial combinations of calcium channel blockers and current antileishmanial drugs. Mem Inst Oswaldo Cruz. 2011;106(8):1032-8. doi: 10.1590/S0074-02762011000800022. [PubMed: 22241129].

19. Aronson NE, Wortmann GW, Johnson SC, Jackson JE, Gasser RJ, Magill AJ, et al. Safety and efficacy of intravenous sodium stibogluconate in the treatment of leishmaniasis: recent U.S. military experience. Clin Infect Dis. 1998;27(6):1457-64. doi:10.1086/515027. [PubMed: 9868660].

20. Domingo P, Ferrer S, Kolle L, Munoz C, Rodriguez P. Acute pancreatitis associated with sodium stibogluconate treatment in a patient with human immunodeficiency virus. Arch Intern Med. 1996;156(9):1029. doi: 10.1001/archinte.1996.00440090141020. [PubMed: 8624172] 1032.

21. Croft SL, Sundar S, Fairlamb AH. Drug resistance in leishmaniasis. Clin Microbiol Rev. 2006;19(1):111-26. doi: 10.1128/CMR.19.1.111-126.2006. [PubMed: 16418526].

22. Sindermann H, Croft SL, Engel KR, Bommer W, Eibl HJ, Unger C, et al. Miltefosine (Impavido): the first oral treatment against leishmania- sis. Med Microbiol Immunol. 2004;193(4):173-80. doi: 10.1007/s00430003-0201-2. [PubMed: 14513375].

23. Ganguly NK. Oral miltefosine may revolutionize treatment of visceral leishmaniasis. TDR news. 2002;68(2).

24. van Griensven J, Balasegaram M, Meheus F, Alvar J, Lynen L, Boelaert M. Combination therapy for visceral leishmaniasis. Lancet Infect Dis. 2010;10(3):184-94. doi: 10.1016/S1473-3099(10)70011-6. [PubMed: 20185097].

25. de Souza W, Sant'Anna C, Cunha-e-Silva NL. Electron microscopy and cytochemistry analysis of the endocytic pathway of pathogenic protozoa. Prog Histochem Cytochem. 2009;44(2):67-124. doi: 10.1016/j.proghi.2009.01.001. [PubMed:19410686].

26. Soares MJ, de Souza W. Endocytosis of gold-labeled proteins and LDL by Trypanosoma cruzi. Parasitol Res. 1991;77(6):461-8. doi: 10.1007/BF00928410. [PubMed: 1656428].

27. Urbina JA. Lipid biosynthesis pathways as chemotherapeutic targets in kinetoplastid parasites. Parasitology. 1997;114 Suppl:S91-9. [PubMed: 9309771].

28. Roberts CW, McLeod R, Rice DW, Ginger M, Chance ML, Goad LJ. Fatty acid and sterol metabolism: potential antimicrobial targets in apicomplexan and trypanosomatid parasitic protozoa. Mol Biochem Parasitol. 2003;126(2):129-42. doi: 10.1016/S0166-6851(02)00280-3. [PubMed: 12615312].

29. Serrano-Martin X, Payares G, De Lucca M, Martinez JC, MendozaLeon A, Benaim G. Amiodarone and miltefosine act synergistically against Leishmania mexicana and can induce parasitological cure in a murine model of cutaneous leishmaniasis. Antimicrob Agents Chemother. 2009;53(12):5108-13. doi: 10.1128/AAC.00505-09. [PubMed: 19805563].

30. Courchesne WE. Characterization of a novel, broad-based fungicidal activity for the antiarrhythmic drug amiodarone.JPharmacol Exp Ther. 2002;300(1):195-9. doi: 10.1124/jpet.300.1.195. [PubMed: 11752116]

31. Gupta SS, Ton VK, Beaudry V, Rulli S, Cunningham K, Rao R. Antifungal activity of amiodarone is mediated by disruption of calcium homeostasis. J Biol Chem. 2003;278(31):28831-9. doi: 10.1074/jbc.M303300200. [PubMed: 12754197].

32. Pozniakovsky AI, Knorre DA, Markova OV, Hyman AA, Skulachev VP, Severin FF. Role of mitochondria in the pheromone- and amiodaroneinduced programmed death of yeast. J Cell Biol. 2005;168(2):257-69. doi: 10.1083 /jcb.200408145. [PubMed: 15657396].

33. Veiga-Santos P, Barrias ES, Santos JF, de Barros Moreira TL, de Carvalho TM, Urbina JA, et al. Effects of amiodarone and posaconazole on the growth and ultrastructure of Trypanosoma cruzi. Int J Antimicrob Agents. 2012;40(1):61-71. doi: 10.1016/j.ijantimicag.2012.03.009. [PubMed: 22591838].

34. Pirson P, Leclef B, Trouet A. Activity of ketoconazole derivatives against Leishmania mexicana amazonensis within mouse peritoneal macrophages. Ann Trop Med Parasitol. 1990;84(2):133-9. doi: 10.1080/00034983.1990.11812446. [PubMed: 2383093].

35. Lazardi K, Urbina JA, de Souza W. Ultrastructural alterations induced by two ergosterol biosynthesis inhibitors, ketoconazole and terbinafine, on epimastigotes and amastigotes of Trypanosoma (Schizotrypanum) cruzi. Antimicrob Agents Chemother. 1990;34(11):2097-105. doi: 10.1128/AAC.34.11.2097. [PubMed: 2073100].

36. Wagner H, Ulrich-Merzenich G. Synergy research: approaching a new generation of phytopharmaceuticals. Phytomedicine. 2009;16(2-3):97110. doi: 10.1016/j.phymed.2008.12.018. [PubMed:19211237] 